\title{
Quasinormal modes for nh-stu black holes
}

\author{
Manavendra Mahato ${ }^{\mathrm{a}}$, Ajay Pratap Singh \\ Indian Institute of Technology Indore, Simrol, Khandwa road, Indore, Madhya Pradesh 453552, India
}

Received: 26 July 2018 / Accepted: 27 September 2018 / Published online: 12 October 2018

(c) The Author(s) 2018

\begin{abstract}
We compute and investigate the behavior of scalar quasinormal frequencies for static, asymptotically anti de Sitter nh-stu black hole solution of $\mathcal{N}=2, D=4$ supergravity. We report their exact expression when the horizon topology is flat. The modes are purely imaginary unless they carry high angular momentum number. These modes for spherical horizon topology were numerically computed by using continued fraction method and found to be of two kinds. One such family consists of purely negative imaginary modes. The other family consists of complex modes with their imaginary part always negative, depicting stability of black holes against scalar perturbations. The complex modes show symmetry about imaginary axis, linear relationship with inverse temperature for large horizon radius and increased oscillation frequency for higher harmonics. Many of these properties are common to a family of black holes which asymptotes to anti de Sitter space, thus pointing to common features of thermalization dynamics of a class of dual holographic theories.
\end{abstract}

\section{Introduction}

Due to unique combination of presence of high curvatures in their geometry and simplicity of many of the known solutions, black holes have remained promising candidates to learn about novel quantum aspects of gravity. One useful such property is given by quasinormal modes in the black hole background. When one perturbs a black hole or its surrounding geometry, the perturbation oscillates like a normal mode of a closed system. These perturbations always decay with the corresponding frequencies usually being complex. A class of them either radiate out to infinity (for asymptotic flat or de Sitter case) or vanish at the boundary (for asymptotic anti de Sitter case). Such black hole oscillations are known as "quasi-normal modes" and they encode important properties

\footnotetext{
a e-mail: manav7up@gmail.com
}

about the dynamics of the black hole. The negative imaginary part of the complex frequencies is inversely proportional to the temperature of black hole. Quasinormal modes dominate the late time behaviour of the dynamics of the black hole akin to hydrodynamic modes. In the later stages of black hole formation, the gravitational waves include certain quasinormal modes that dominate the emission.

Quasinormal modes of black holes have been an interesting topic of discussion because they are those characteristics of black holes which do not depend on initial perturbations and are functions of black hole parameters only. This means that quasinormal modes encode unique characteristic features which hopefully can lead to the direct identification of the black hole existence. Recent interest in quasinormal modes of black holes arose since these quasi-normal frequencies are relevant to experiments for detecting gravitational waves such as the recent detection by LIGO of these waves emanating from colliding black holes [1]. The gravitational waves can also be emitted from supernovae or coalescence of binary neutron stars, which are thought to eventually form a black hole. Such waves, at late times, can have frequencies or features similar to those calculated for quasinormal modes. In general, quasinormal modes are important in black holes dynamics and appear in processes such as birth of black holes, collisions of two black holes, decay of different fields in a black hole background, etc [2-4].

Another source of interest in quasinormal modes is due to existence of a correspondence between asymptotic anti de Sitter gravity and quantum field theory in flat space-time, widely known as gauge/gravity correspondence [5]. According to this correspondence, a large static black hole in asymptotically AdS spacetime corresponds to an approximately thermal state of conformal field theory. The perturbation in the black hole corresponds to perturbation in thermal state, and the decay of the perturbation describes the return to the thermal equilibrium. So we can obtain a prediction for thermalization time scale in the strongly coupled field theory by introducing a perturbation in holographically dual AdS black 
hole solution which will ultimately evolve according to the quasi-normal frequencies. Thus, the quasinormal mode of an AdS black hole has an interpretation as a time scale for the approach to thermal equilibrium. Moreover, these modes are also related to Green's function of appropriate operator corresponding to the perturbation in holographically dual field theories. There, the poles of the Green's function are the quasi-normal frequencies. Thus, quasinormal modes using this duality has led to important progress in our understanding of the physics of a class of gauge theories [6-9].

An asymptotic anti de Sitter black hole in gauged supergravity theories provides a dual description of certain strongly coupled non abelian quantum field theory at finite temperature. Due to inherent symmetries, these theories are more amenable to calculations and have been well studied in literature as a representative of black holes in presence of various matter. For more richer situations, we choose in this article to calculate quasi-normal modes of scalar perturbations in the background of BPS black hole solution in $\mathcal{N}=2, D=4$ Fayet-Iliopoulos gauged supergravity theories which contain certain scalar potential and a non-homogenous special Kahler manifold parameterized by the vector multiplet scalars [10]. The solution is referred as a non-homogeneous deformation of stu model (nh-stu). The remainder of the paper is organized as follows. In Sect. 2, we briefly review the nh-stu black hole solution of $\mathcal{N}=2, D=4$ supergravity. In Sect. 3, we drive the scalar field equation in this black hole background. We solve it to get exact quasinormal modes for flat horizon topology in Sect. 4. In Sect. 5, we analyze the field equation for the case with spherical horizon topology to express an implicit equation for quasinormal modes using modified version of continued fraction method [11-14]. Numerical results from computation of quasinormal modes and their behavior are discussed in Sect. 5.1. The concluding Sect. 6 contains some outlook and scope for future studies.

\section{Review of $\mathcal{N}=2, D=4$ supergravity solution}

We present a brief description in this section of a solution of $\mathcal{N}=2, D=4$ supergravity coupled to $n_{V}$ Abelian vector multiplets. More details are available in original paper [10]. We represent the space time indices by greek letters. The bosonic field content consists of a veilbein $e_{\mu}^{a}, n_{V}+1$ vector potentials denoted as $A_{\mu}^{\Lambda}$ with $\Lambda=0, \ldots, n_{V}$ and $n_{V}$ complex scalars denoted as $z^{i}$ with $i=1, \ldots, n_{V}$. The vector potential $A_{\mu}^{0}$ denotes graviphoton while the rest of them are components of vector supermultiplets.

The scalar moduli space is a special Kahler manifold. It is a symplectic bundle over a base of $n_{V}$ dimensional Hodge Kahler manifold with covariant holomorphic sections

$\mathcal{V}=\left(\begin{array}{c}L^{\Lambda} \\ M_{\Lambda}\end{array}\right)$.
Kahler covariant derivative is denoted as

$\mathcal{D}_{i} \mathcal{V}=\partial_{i} \mathcal{V}-\frac{1}{2}\left(\partial_{i} \mathcal{K}\right) \mathcal{V}$

Here, $\mathcal{K}$ is the Kahler potential. A symplectic inner product constraint $i\langle\mathcal{V}, \mathcal{V}\rangle=1$ restricts the section $\mathcal{V}$ which can also be written in terms of a holomorphic symplectic vector as

$\mathcal{V}=e^{\mathcal{K} / 2}\left(\begin{array}{c}X^{A} \\ F_{A}\end{array}\right)$.

The couplings between vectors and scalars are given by a field dependent matrix $\mathcal{N}_{\Lambda \Sigma}=\mathcal{R}_{\Lambda \Sigma}+i \mathcal{I}_{\Lambda \Sigma}$, where $\mathcal{R}_{\Lambda \Sigma}$ and $\mathcal{I}_{\Lambda \Sigma}$ are real and imaginary parts of $\mathcal{N}_{\Lambda \Sigma}$. The relation is written as

$M_{\Lambda}=\mathcal{N}_{\Lambda \Sigma} L^{\Sigma}$ and $D_{i} \bar{M}_{\Lambda}=\mathcal{N}_{\Lambda \Sigma} D_{i} \bar{L}^{\Sigma}$.

One also constructs a superpotential $\mathcal{L}$ using a symplectic inner product between Fayet-Illioupoulos parameters $\mathcal{G}=$ $\left(g^{\Lambda}, g_{\Lambda}\right)$ and symplectic vector $\mathcal{V}$ as $\mathcal{L}=\langle\mathcal{G}, \mathcal{V}\rangle$. The scalar potential is given in terms of the superpotential as $V_{g}=$ $g^{i} \bar{j} D_{i} \mathcal{L} \bar{D}-\bar{j} \overline{\mathcal{L}}-3|\mathcal{L}|^{2}$.

The bosonic Lagrangian is

$$
\begin{aligned}
\mathcal{L}= & \frac{R}{2}-g_{i j} \partial_{\mu} z^{i} \partial^{\mu} \bar{z}^{j}+\frac{1}{4} \mathcal{I}_{\Lambda \Sigma} F_{\mu \nu}^{\Lambda} F^{\Sigma \mu \nu} \\
& +\frac{1}{8 \sqrt{-g}} \epsilon^{\mu \nu \rho \sigma} \mathcal{R}_{\Lambda \Sigma} F_{\mu \nu}^{\Lambda} F_{\rho \sigma}^{\Sigma}-V_{g} .
\end{aligned}
$$

A metric ansatz for a static and spherically symmetric black hole is taken in [10],

$d s^{2}=-e^{2 U(r)} d t^{2}+e^{-2 U(r)}\left(d r^{2}+e^{2 \psi(r)} d \Omega_{\kappa}^{2}\right)$,

where $d \Omega_{\kappa}^{2}=d \theta^{2}+f_{\kappa}^{2}(\theta) d \phi^{2}$ is a constant curvature 2D metric with $f_{\kappa}=(\sin \theta, \theta, \sinh \theta)$ for hyperbolic, flat and spherical metrics denoted by $\kappa=(1,0,-1)$ respectively. The solution can be written containing a metric

$$
\begin{aligned}
d s^{2} & =-e^{2 U(r)} d t^{2}+e^{-2 U(r)}\left(d r^{2}+e^{2 \psi(r)} d \Omega_{k}^{2}\right), \\
e^{2 U(r)} & =\frac{\left(a r^{2}-c_{1}\right)^{2}}{l_{A}^{2}\left(a r-c_{4}\right) \sqrt{\left(a r+2 c_{2}\right)\left(a r+2 c_{3}\right)}}, \\
e^{2 \psi} & =\left(a r^{2}-c_{1}\right)^{2},
\end{aligned}
$$

where $l_{A}$ is the radius of the asymptotic AdS metric which will be taken to be unity from now onwards [10]. The constants $c_{1}, c_{2}, c_{3}$ and $c_{4}$ can be expressed in terms of gauge couplings, charges and a parameter denoting nonhomogeneity. Moreover, $c_{4}=c_{2}+c_{3}$ and constant $a$ can be set to unity by scaling radial variable. The above solution represents a black hole, with a horizon at $r_{0}=\sqrt{c_{1} / a}$. Three charges and FI parameters together satisfy Dirac quantization condition given as $g_{0} p^{0}-g^{i} p_{i}=-\kappa$. In terms of the parameters chosen here, this constraint assumes the form

$3 c_{2}^{2}+3 c_{3}^{2}+2 c_{2} c_{3}-2 r_{0}^{2}=-\kappa$. 
The topology of horizon can be either spherical, flat or hyperbolic, corresponding to the value of $\kappa=(1,0,-1)$. In the asymptotic limit $r \rightarrow \infty$, the metric in Eq. (7) becomes $A d S_{4}$. On the other hand, when $\mathrm{r}$ approaches the horizon $r_{0}$, the spacetime becomes $A d S_{2} \times \Sigma$. The metric contains three free parameters and full general analysis will be tedious. However, one can obtain some simplification by looking at a particular subset of the parameter space where $c_{4}=2 c_{2}=2 c_{3}$. We will focus on this representative metric among the above family of metrics given in Eq. (7) in later sections. The metric now reduces to

$d s^{2}=-\frac{\left(r^{2}-r_{0}^{2}\right)^{2}}{\left(r^{2}-c_{4}^{2}\right)} d t^{2}+\frac{\left(r^{2}-c_{4}^{2}\right)}{\left(r^{2}-r_{0}^{2}\right)^{2}} d r^{2}+\left(r^{2}-c_{4}^{2}\right) d \Omega_{\kappa}^{2}$.

Dirac quantization condition now determines the parameter $c_{4}$ in terms of horizon radius and curvature of horizon,

$c_{4}=\sqrt{\frac{1}{2}\left(2 r_{0}^{2}-\kappa\right)}$.

The black hole in Eq. (9) has a temperature $T^{-1}=2 \pi r_{0}(1-$ $B$ ), where $B$ denotes $\frac{c_{4}^{2}}{r_{0}^{2}}$.

\section{The scalar field equation}

We further explore the solution in previous section by studying scalar perturbations in its background. Perturbations of gravity solutions are very important to study their intrinsic properties, such as their natural frequencies and to test their stability against such perturbations. The study of perturbations of gravitational objects, such as a black hole is closely linked to the gravitational wave emission. The perturbations are falling into the black hole at the horizon and are outgoing at the boundary for asymptotic flat spacetimes. Since the potential for asymptotic AdS space time rises exponentially near the boundary, the perturbation modes vanish there for such cases. Each frequency of emission spectrum is usually complex whose real part tells about oscillations of the perturbations and the imaginary part is related to the damping timescale. These modes are called quasi-normal modes. The simplest such perturbations are scalar perturbations. In order to compute the quasinormal modes associated with decay of the scalar field around our 4 dimensional nh-stu black hole, we must solve the Klein Gordon equation for scalar expressed as

$\left[\frac{1}{\sqrt{-g}} \partial_{\mu}\left(\sqrt{-g} g^{\mu \nu} \partial_{\nu}\right)-m^{2}\right] \Phi=0$,

where the determinant of metric (7) is,

$\sqrt{-g}=e^{(2 \psi(r)-2 U(r))} f_{\kappa}(\theta)$.
Thus, we get the following scalar field equation for our black hole background,

$$
\begin{gathered}
\partial_{r}\left[e^{2 \psi(r)} \partial_{r} \Phi\right]-e^{(2 \psi(r)-4 U(r))} \partial_{t}^{2} \Phi \\
+\frac{1}{f_{\kappa}(\theta)} \partial_{\theta}\left[f_{\kappa}(\theta) \partial_{\theta} \Phi\right]+\frac{1}{f_{\kappa}^{2}(\theta)} \partial_{\phi}^{2} \Phi \\
-m^{2} e^{(2 \psi(r)-2 U(r))} \Phi=0,
\end{gathered}
$$

where we have rearranged the terms so that the separability of the equation is apparent. This form, along with the spherical symmetry and time independence of the metric, suggests the following decomposition of the solution in terms of radial and angular variables,

$\Phi=e^{-i \omega t} R(r) Y_{m}^{l}(\theta, \phi)$.

Here, $Y_{m}^{l}(\theta, \phi)$ are spherical harmonics and $l$ denotes the angular momentum number. The radial part of the field equation turns out to be,

$$
\begin{aligned}
& \partial_{r}\left[e^{2 \psi(r)} \partial_{r} R\right]+\left[\omega^{2} e^{(2 \psi(r)-4 U(r))}\right. \\
& \left.\quad-l(l+1)-m^{2} e^{(2 \psi(r)-2 U(r))}\right] R=0 .
\end{aligned}
$$

After substitution of the values of metric coefficients, the equation reduces to

$$
\begin{gathered}
\frac{d^{2} R}{d r^{2}}+\frac{4 r}{\left(r^{2}-r_{0}^{2}\right)} \frac{d R}{d r}+\left[\frac{\omega^{2}\left(r^{2}-c_{4}^{2}\right)^{2}}{\left(r^{2}-r_{0}^{2}\right)^{4}}\right. \\
\left.-\frac{l(l+1)}{\left(r^{2}-r_{0}^{2}\right)^{2}}-m^{2} \frac{\left(r^{2}-c_{4}^{2}\right)}{\left(r^{2}-r_{0}^{2}\right)^{2}}\right] R=0 .
\end{gathered}
$$

It will be convenient for later analysis to map the entire region of interest $\left(r_{0}<r<\infty\right)$, into a finite parameter range. In order to do so, we change the variable to $z=1-\frac{r_{0}^{2}}{r^{2}}$, resulting in

$$
\begin{aligned}
& 4 z(1-z) \frac{d^{2} R}{d z^{2}}+2(4-3 z) \frac{d R}{d z} \\
& +\frac{1}{r_{0}^{2}}\left[\frac{\kappa^{2}(z) \omega^{2}}{z^{3}}-\frac{l(l+1)}{z}-\frac{m^{2} r_{0}^{2} \sigma(z)}{z(1-z)}\right] R=0,
\end{aligned}
$$

where $\sigma(z)=(1-B)+B z$. The new dimensionless variable $z$ vanishes at the horizon and approaches unity at the boundary. We need to take into account horizon topology for further analysis.

\section{Quasinormal modes for flat horizon topology}

We consider here the case when the geometry along two transverse spacelike directions is a plane. This subspace is conformally flat and horizon of the black hole has flat topology. This is also denoted by $\kappa=0$ case in Sect. 2. One obtains additional simplification of the scalar equation in this case 
so that one can analyze the problem analytically and obtain exact expressions for the quasinormal modes even with non trivial scalar mass. In this section, we elaborate in this direction. The constant $c_{4}$ simplifies to be equal to the horizon radius $r_{0}$. The constant $B$ in the differential equation reduces to unity. The scalar field equation for general mass simplifies to become

$$
\begin{aligned}
& 2 z^{2}(1-z) \frac{d^{2} R}{d z^{2}}+z(4-3 z) \frac{d R}{d z} \\
& +\left[\frac{\omega^{2}-l(l+1)}{2 r_{0}^{2}}-\frac{m^{2} z}{2(1-z)}\right] R=0 .
\end{aligned}
$$

It has regular singular points at 0,1 and $\infty$ and hence, it offers exact solution in terms of hypergeometric function.

$R(z)=z^{\alpha}(1-z)^{s-\alpha} F\left[s, s+\frac{1}{2}, 2 \alpha+2, z\right]$,

where,

$$
\begin{aligned}
& \alpha=-\frac{1}{2}-\frac{i}{2 r_{0}} \sqrt{\omega^{2}-l(l+1)-r_{0}^{2}}, \\
& s=\frac{1}{4}+\frac{\sqrt{4 m^{2}+9}}{4}-\frac{i}{2 r_{0}} \sqrt{\omega^{2}-l(l+1)-r_{0}^{2}} .
\end{aligned}
$$

The above solution is ingoing at the horizon. The conjugate of the above solution will be another independent solution of the differential equation. Since it is outgoing at the horizon, we can neglect it and focus on the solution in Eq. (19) above. We require the solution in (19) to be vanishing at the boundary for quasinormal frequencies. Hence, we expand the solution at boundary using an identity satisfied by hypergeometric function. ( See for example, (15.3.6) of [15].)

$$
\begin{aligned}
& R(z) \sim z^{\alpha}(1-z)^{s-\alpha} \frac{\Gamma[2 \alpha+2] \Gamma[2 \alpha-2 s-3 / 2]}{\Gamma[a] \Gamma[a-1 / 2]} \\
& \quad \times F\left[s, s+\frac{1}{2}, 2 s-2 \alpha-\frac{1}{2}, 1-z\right] \\
& \quad+z^{\alpha}(1-z)^{\alpha-s+3 / 2} \frac{\Gamma[2 \alpha+2] \Gamma[2 s-2 \alpha-3 / 2]}{\Gamma[s] \Gamma[s+1 / 2]} \\
& \quad \times F\left[a, a-\frac{1}{2}, 2 \alpha-2 s+\frac{5}{2}, 1-z\right],
\end{aligned}
$$

where, $a=2 \alpha+2-s$. The asymptotic behavior at the boundary of the two parts of the above solution determines that the former is a non normalizable mode and the latter is a normalizable mode. The latter mode vanishes as $(1-z)^{-\beta}$, with $\beta=\left(\sqrt{4 m^{2}+9}-9\right) / 4$ as one approaches boundary. To satisfy the quasinormal mode boundary conditions, the whole solution should vanish at the boundary. So we need the coefficients of non normalizable mode to vanish, which occurs if either $2 \alpha-s+2+n=0$ or $2 \alpha-s+n+\frac{3}{2}=0$ for $n \geq 0$ is satisfied. It results in the Gamma function in the denominator of the coefficient of the non normalizable mode to diverge. These two conditions can together be written as

$$
\begin{aligned}
& 4 \alpha-2 s+3+n=0 \text { for } n \geq 0, \\
& \text { i.e. } \frac{2 i}{r_{0}} \sqrt{\omega^{2}-l(l+1)-r_{0}^{2}}=2 n+1-\sqrt{4 m^{2}+9} .
\end{aligned}
$$

It can be rewritten to obtain an expression for quasinormal frequency as

$$
\omega^{2}=l(l+1)+r_{0}^{2}-\frac{r_{0}^{2}}{4}\left[2 n+1-\sqrt{4 m^{2}+9}\right]^{2},
$$

with $\omega$ being the positive imaginary root of the above expression. Hence, we observe that in this peculiar case, any quasinormal mode is going to be purely imaginary. Another peculiarity is that one can get real $\omega$ for large angular momentum number. Such modes are purely oscillating at the horizon and shouldn't contribute to decay of the perturbations. Since the asymptotic metric is anti de Sitter space, we can think of boundary value of scalar field acting as a source for perturbations for certain operator $\mathcal{O}$ in the dual field theory in the context of gauge/gravity correspondence. The form of two point Green's function of the dual operator can be easily calculated from the expression (21) to be

$G[\mathcal{O}, \mathcal{O}] \sim \frac{\Gamma[a] \Gamma[a-1 / 2] \Gamma[2 s-2 \alpha-3 / 2]}{\Gamma[s] \Gamma[s+1 / 2] \Gamma[2 \alpha-2 s-3 / 2]}$.

Hence, the quasinormal modes can also be interpreted as poles of the above Green's function [3,4,16,17].

\section{Quasinormal modes for spherical horizon topology}

We focus in this section on the case when a two sphere metric is transverse to radial direction. It is also referred as $\kappa=1$ in section (2). Since the quasinormal modes represent decay of the perturbations in the gravity background, the dominant ones will be those that can be easily excited. We will hence restrict this study to massless modes i.e. we take $m=0$. The radial equation reduces to

$$
\frac{d}{d r}\left[e^{2 \psi(r)} \frac{d R}{d r}\right]+\left[\omega^{2} e^{(2 \psi(r)-4 U(r))}-l(l+1)\right] R=0 .
$$

The above Eq. (17) is a second-order ordinary differential equation with only one irregular singularity at $z=0$, which makes the series expansion about the horizon divergent. So, it does not offer solution using Frobenius method. We further investigate it for its asymptotic behavior.

1. Behavior near horizon: As we approach horizon, $r \rightarrow$ $r_{0}, x \rightarrow 1$ and $z \rightarrow 0$. One can assume the solution near the boundary as $R(r)=\exp [\psi(r)]$ inspired by WKB method to find solution and obtain non linear equation for $\psi(r)$. 
Expanding $\psi(r)$ in series and keeping the leading terms and appropriate sign leads to

$R \rightarrow z^{-\frac{i}{4}(1+B) \omega} \exp \left(\frac{i(1-B) \omega}{2 z}\right)(1+\cdots)$.

The sign is chosen so that the field radiate only in inward direction at the horizon. The horizon radius $r_{0}$ is set to unity by redefining $\omega$ and $l$.

2. Behavior near boundary,

Near boundary, $r \rightarrow \infty, x \rightarrow 0$ and $z \rightarrow 1$. The boundary $z=1$ is a regular singularity of the equation, thus latter admits a power series solution near the boundary,

$R \rightarrow(1-z)^{3 / 2}(1+\cdots)$.

Appropriate sign is chosen above so that the solution decays at infinity.

In order to impose both the requisite boundary conditions, we consider the following expansion of the solution

$R=(1-z)^{3 / 2} z^{-\frac{i(1+B) \omega}{4}} e^{\frac{i(1-B) \omega}{2 z}} \sum_{n=0}^{\infty} a_{n}(1-z)^{n}$

The quasi-normal mode boundary condition are satisfied if and only if $\sum a_{n}(1-z)^{n}$ converges at both boundaries. To examine the convergence of above summation, we first obtain the recurrence relation of coefficients $a_{n}$ of the Eq. (28). To do so, we substitute the above series expansion (28) into Eq. (17) and we obtain a three term recurrence relation:

$$
\begin{aligned}
& \alpha_{0} a_{1}+\beta_{0} a_{0}=0, \\
& \alpha_{n} a_{n+1}+\beta_{n} a_{n}+\gamma_{n} a_{n-1}=0 .
\end{aligned}
$$

Here, the recurrence coefficients are,

$$
\begin{aligned}
\alpha_{n}= & -8(n+1)(2 n+5) \\
\beta_{n}= & \left(32 n^{2}-32 n+48\right)+4 l+4 l^{2}-30 i \omega+10 i B \omega \\
& -4 \omega^{2}+n(112-24 i \omega+8 i B \omega) \\
\gamma_{n}= & -\left(16 n^{2}-48 n+80\right)+14 i \omega+14 i B \omega+\omega^{2} \\
& +2 B \omega^{2}+B^{2} \omega^{2}+8(n-1) i(9 i+\omega+B \omega)
\end{aligned}
$$

The recurrence coefficients $\alpha_{n}, \beta_{n}$ and $\gamma_{n}$ are simple functions of $n$ and the parameters $(l, B$ and $\omega)$ of the differential equation.

One possible way to test convergence of the power series in (28) at both the boundaries is to analyze the large- $n$ behavior of $\frac{a_{n+1}}{a_{n}}$.

The ratio of the successive $a_{n}$ will be given by the infinite continued fraction,

$$
\frac{a_{n+1}}{a_{n}}=-\frac{\gamma_{n+1}}{\beta_{n+1}-\frac{\alpha_{n+1} \gamma_{n+2}}{\beta_{n+2}-\frac{\alpha_{n+2} \gamma_{n+3}}{\beta_{n+3}-\cdots}}}
$$

We obtain a characteristic equation for quasi-normal frequencies by evaluating (31) at $n=0$,

$$
\frac{a_{1}}{a_{0}}=-\frac{\gamma_{1}}{\beta_{1}-\frac{\alpha_{1} \gamma_{2}}{\beta_{2}-\frac{\alpha_{2} \gamma_{3}}{\beta_{3}-\cdots}}}
$$

Alternatively, the above ratio can also be determined using Eq. (29) to be

$\frac{a_{1}}{a_{0}}=-\frac{\beta_{0}}{\alpha_{0}}$.

Using the above two relations, one obtains the desired characteristic equation,

$$
\beta_{0}-\frac{\alpha_{0} \gamma_{1}}{\beta_{1}-\frac{\alpha_{1} \gamma_{2}}{\beta_{2}-\frac{\alpha_{2} \gamma_{3}}{\beta_{3}-\cdots}}}=0 .
$$

The roots of the above equation will be the sought after quasinormal frequencies.

\subsection{Numerical results}

To determine the quasi-normal frequencies we have to find the roots of the desired characteristic equation (34) which involves an infinite continued fraction whose elements are functions of the frequency. The suitable frequency satisfying them can be found numerically using Newton-Raphson rootfinding algorithm. ${ }^{1}$ The elements of the infinite continued fraction are functions of $\omega$ and we can get more number of quasi-normal frequencies as roots by increasing the number of terms in the continued fraction. The characteristic equation have an infinite number of terms, so we need to make an approximation by truncating it to certain number of terms before looking for the roots. So, for any desired accuracy, one needs to find the roots of the equation by increasing the number of terms in the truncated continued fraction. For a root with lowest imaginary value, we plot a graph between its imaginary part vs number of iterations.

From the Fig. 1, we can see the quasi-normal modes are fast converging with increasing the number of iterations. We estimate the convergence to be exponential and fit these converging data as a function of number of iterations $(n)$,

$\omega=a+b \exp \left(\frac{-n}{c}\right)$

where, $a=-0.448261, b=-3.75736$ and $c=8.51034$ with goodness of fit parameter $\chi^{2}=.00083$. Lower the value

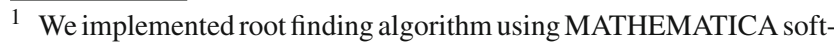
ware.
} 


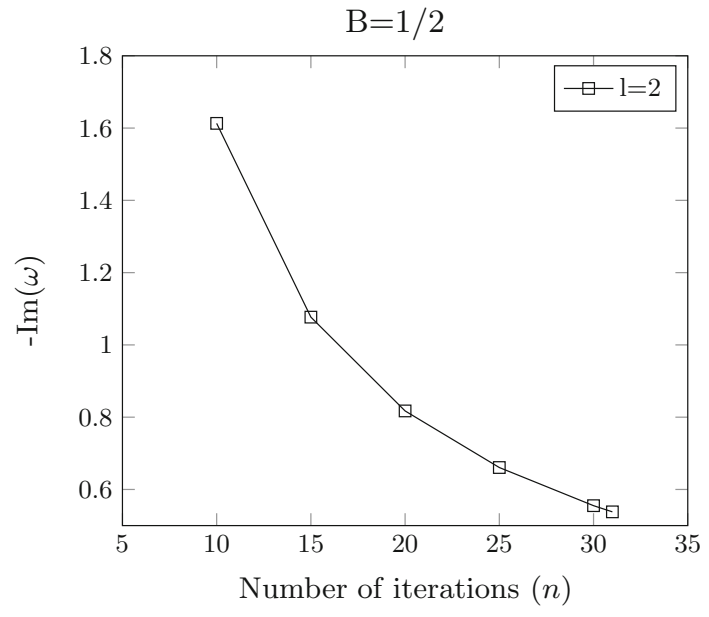

Fig. $1 \omega$ with $n$ for $B=1 / 2$

Table 1 Quasi-normal frequencies for $\mathrm{B}=1 / 2$

\begin{tabular}{cccccccc}
\hline 1 & $\mathrm{n}$ & $\operatorname{Re}(\omega)$ & $\operatorname{Im}(\omega)$ & 1 & $\mathrm{n}$ & $\operatorname{Re}(\omega)$ & $\operatorname{Im}(\omega)$ \\
\hline 0 & 25 & 0.8573 & -3.1686 & 2 & 25 & 2.3539 & -2.6727 \\
& 26 & 0.8640 & -3.1364 & & 26 & 2.3541 & -2.6730 \\
& 27 & 0.8609 & -3.1079 & & 27 & 2.3544 & -2.6732 \\
& 28 & 0.8503 & -3.0840 & & 28 & 2.3547 & -2.6732 \\
& 39 & 0.8337 & -3.0652 & & 29 & 2.3548 & -2.6731 \\
& 30 & 0.8120 & -3.0652 & & 30 & 2.3550 & -2.6729 \\
1 & 25 & 1.3909 & -2.8650 & 3 & 25 & 3.3729 & -2.5954 \\
& 26 & 1.3984 & -2.8665 & & 26 & 3.3729 & -2.5954 \\
& 27 & 1.4045 & -2.8648 & & 27 & 3.3729 & -2.5954 \\
& 28 & 1.4086 & -2.8612 & & 28 & 3.3729 & -2.5954 \\
& 29 & 1.4105 & -2.8569 & & 29 & 3.3729 & -2.5954 \\
& 30 & 1.4106 & -2.8525 & & 30 & 3.3729 & -2.5954 \\
& & & & & & &
\end{tabular}

of $c$, the faster will be the convergence, thus requiring lower number of terms for any given desired accuracy. We also list the values in Table 1.

From the table, we can say that for each value of 1 after taking 30 number of terms in the continued fraction, quasinormal frequency is becoming constant up to two decimal points. So we decided to truncate with 30 number of terms for further results and calculations. One notable aspect of our calculation is that we also obtained some purely imaginary modes as quasinormal frequencies even using continued fraction method. We list them in Table 2 . The possibility of such modes in other simpler situations has been reported in literature $[18,19]$.

Our characteristic equation has two parameters $l$ and $B$. So we computed first ten lowest order quasi-normal modes from $l=0$ to $l=5$ with $B=1 / 2$, which are shown explicitly in Fig. 2. Here, the size of the black hole is fixed to unity, i.e. $\left(r_{0}=1\right)$.
Table 2 Imaginary quasi-normal frequencies for $\mathrm{B}=1 / 2$

\begin{tabular}{|c|c|c|c|c|c|}
\hline 1 & $\operatorname{Re}(\omega)$ & $\operatorname{Im}(\omega)$ & 1 & $\operatorname{Re}(\omega)$ & $\operatorname{Im}(\omega)$ \\
\hline \multirow[t]{8}{*}{0} & 0.0 & -0.23559 & \multirow[t]{8}{*}{3} & 0.0 & -0.80073 \\
\hline & 0.0 & -0.80522 & & 0.0 & -1.85155 \\
\hline & 0.0 & -1.77237 & & 0.0 & -3.55538 \\
\hline & 0.0 & -4.40241 & & 0.0 & -6.9749 \\
\hline & 0.0 & -26.8484 & & 0.0 & -25.6236 \\
\hline & 0.0 & -36.5261 & & 0.0 & -35.9239 \\
\hline & 0.0 & -54.2701 & & 0.0 & -53.9987 \\
\hline & 0.0 & -45.2377 & & 0.0 & -44.8469 \\
\hline \multirow[t]{8}{*}{1} & 0.0 & -0.35889 & \multirow[t]{8}{*}{4} & 0.0 & -1.09163 \\
\hline & 0.0 & -1.04091 & & 0.0 & -2.34747 \\
\hline & 0.0 & -2.23218 & & 0.0 & -4.33724 \\
\hline & 0.0 & -4.90604 & & 0.0 & -8.41743 \\
\hline & 0.0 & -26.656 & & 0.0 & -24.6883 \\
\hline & 0.0 & -36.4274 & & 0.0 & -35.5081 \\
\hline & 0.0 & -54.2251 & & 0.0 & -53.8163 \\
\hline & 0.0 & -45.1731 & & 0.0 & -44.5822 \\
\hline \multirow[t]{8}{*}{2} & 0.0 & -0.55516 & \multirow[t]{8}{*}{5} & 0.0 & -1.42751 \\
\hline & 0.0 & -1.41471 & & 0.0 & -2.9055 \\
\hline & 0.0 & -2.86867 & & 0.0 & -5.22298 \\
\hline & 0.0 & -5.82491 & & 0.0 & -10.285 \\
\hline & 0.0 & -26.2581 & & 0.0 & -23.306 \\
\hline & 0.0 & -36.228 & & 0.0 & -34.9706 \\
\hline & 0.0 & -54.1348 & & 0.0 & -53.5865 \\
\hline & 0.0 & -45.0432 & & 0.0 & -44.2464 \\
\hline
\end{tabular}

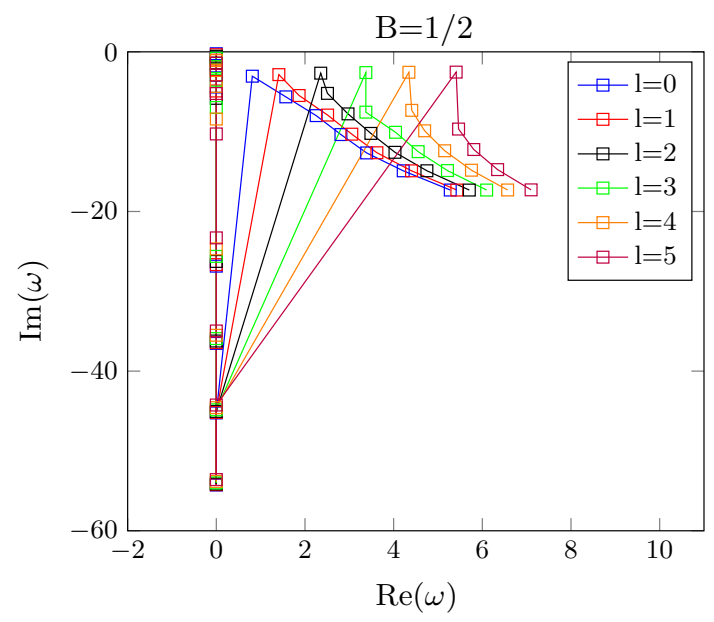

Fig. 2 First 15 quasi-normal mode for $l=0$ to 5 and $\mathrm{B}=1 / 2$

Next, we calculated first 45 quasi-normal frequencies for a representative case of $B=\frac{1}{2}, l=2$ and plotted a graph between real vs imaginary parts of the frequencies. Finally we found a symmetry about the imaginary $\omega$-axis. This feature is a common feature for quasinormal modes in AdS black holes [11,12] (see Fig. 3). 


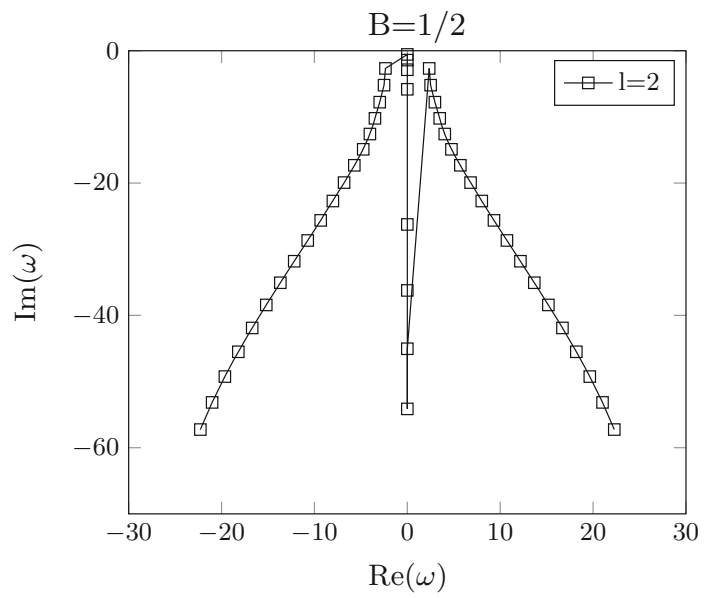

Fig. 3 First 45 quasi-normal frequencies for $1=2$ and $B=1 / 2$

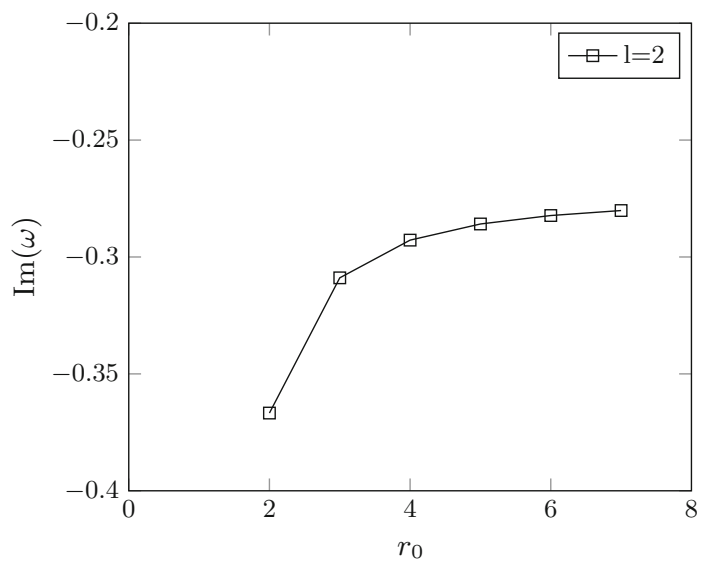

Fig. 4 Size of horizon vs Imaginary part of quasi-normal frequencies for $1=2$

Behavior of quasinormal modes while increasing the size of the black hole can be studied by changing $r_{0}$ which in our case is related to change in parameter $c_{4}$ or $B$. We plotted a graph between size of the black hole $\left(r_{0}\right)$ and the quasinormal frequencies in Fig. 4. This also depicts the variation of the mode with temperature which is inversely proportional to the size of the black hole horizon.

From the above figure, we can say that the imaginary part of the quasi-normal frequency of the large size black hole is the linear function of $r_{0}$ and this linear relation doesn't apply for the intermediate size of the black hole [20]. In this aspect, we find the black hole in our case behaves very similar to AdS black holes [6,11,12,21-25].

\section{Conclusion}

In this article, we have calculated quasinormal modes for nhstu black hole solution of $\mathcal{N}=2, D=4$ supergravity. The calculation is amenable for a particular constraint on charges and gauge couplings given as $c_{2}=c_{3}$ in terms of parameters defined in Sect. 2. In this case, the scalar perturbation equation contains only 3 singularities. For the case of flat horizon topology, it can be exactly solved in terms of hypergeometric functions. These solutions lead to two classes of quasinormal modes, one being purely imaginary. Higher angular momentum numbers in comparison with given scalar mass and horizon radius can result in other class of purely real modes.

We also explored massless scalar perturbations for compact black hole solution of above kind with spherical horizon topology. We proved by adapting an argument for AdS black holes [6] that all quasinormal modes here will have non vanishing negative imaginary component. (See appendix A). So, they all can be interpreted as decaying, thus rendering the black hole stable against any massless scalar perturbation. Furthermore, the quasinormal modes obtained here can be divided into two types, one are purely imaginary negative modes. Higher angular momentum number contributes to faster decay of any such mode. The second kind of modes are complex frequencies with negative imaginary part. Here, the effect of increasing the angular momentum number contributes to shriller oscillations of the mode given by higher magnitude of real part of quasinormal frequencies. These complex modes come in pairs, i.e. for any given quasinormal mode, its negative conjugate is also another mode. Graphically, it leads to quasinormal modes placed symmetrically in the lower half of complex $\omega$ plane. This complex conjugate symmetry of the roots is in consonance with the reflection principle in scattering, when quasinormal modes are interpreted as singularities of the scattering amplitude for an effective gravitational potential felt by traveling perturbations [26-29]. When behavior of a quasinormal frequency with respect to horizon radius is investigated for given angular momentum number, we found a linear relationship for larger size of black holes. This relationship breaks down for intermediate size. Reducing horizon size leads to proportionate increase in the temperature of Hawking radiation. At intermediate sizes, one is likely to encounter a Hawking-Page transition [30], which depicts instability of anti de Sitter black holes preferring thermal gas in anti de Sitter space. Patterns of quasinormal modes do encode effects of phase transitions in many situations [31]. Most of the patterns obtained here for complex quasinormal frequencies are common with those obtained for simpler setting of AdS-Schwarzschild black holes, pointing to very general properties. This points towards general characteristics about dynamics of scalar hydrodynamic modes in holographically dual strongly coupled gauge theories [4]. Full spectrum of quasinormal modes also paves a way to find one loop partition function determinant in such theories $[32,33]$. 
Acknowledgements This research is partly supported by DST-SERB grant no. SR/FTP/PS-149/2011.

Open Access This article is distributed under the terms of the Creative Commons Attribution 4.0 International License (http://creativecomm ons.org/licenses/by/4.0/), which permits unrestricted use, distribution, and reproduction in any medium, provided you give appropriate credit to the original author(s) and the source, provide a link to the Creative Commons license, and indicate if changes were made.

Funded by SCOAP ${ }^{3}$.

\section{Appendix A}

We present here an argument to show that for our black hole with spherical horizon topology $(\kappa=1)$, the imaginary part of the quasinormal frequencies are always negative. This calculation is an adaptation of similar calculation in [6] for our case. According to the boundary condition for the quasinormal mode, the field radiates in only inward direction at the horizon and the solution at the horizon is given in Eq. (26),

$R \rightarrow z^{-\frac{i}{4} \omega(1+B)} \exp \left(\frac{i \omega(1-B)}{2 z}\right)$

The solution must decay in time and it is only possible if the imaginary part of the $\omega$ is negative, i.e., the condition for stable mode is $\operatorname{Im}(\omega)<0$. To prove the condition for any mode, we consider the following form for the solution,

$R(z)=\exp \left(\frac{i \omega(1-B)}{2 z}\right) X(z)$

Let us also denote the function $\frac{z^{2}}{\sqrt{1-z}}$ by $f$. Then Eq. (25) becomes

$$
\begin{aligned}
& {\left[f X_{z}\right]_{z}-\frac{2 b X_{z}}{\sqrt{1-z}}+X\left[\omega^{2} \eta(z)-\frac{b}{2(1-z)^{3 / 2}}\right.} \\
& \left.-\frac{l(l+1)}{4(1-z)^{3 / 2}}\right]=0,
\end{aligned}
$$

where $b=\frac{i \omega(1-B)}{2}$ and

$\eta(z)=\frac{1}{4 r_{0}^{2} z^{2} \sqrt{1-z}}\left(\frac{z}{1-z}+B(1-B)\right)$.

Multiplying (A3) by $X^{*}$ and integrating from $z=0$ to $z=1$ results in

$$
\begin{gathered}
\int_{0}^{1} d z\left[-f\left|X_{z}\right|^{2}-\frac{2 b}{\sqrt{1-z}} X^{*} X_{z}+|X|^{2}\left\{\omega^{2} \eta(z)\right.\right. \\
\left.\left.-\frac{b}{2(1-z)^{3 / 2}}-\frac{l(l+1)}{4(1-z)^{3 / 2}}\right\}\right]=0 .
\end{gathered}
$$

We note that for $0<z<1$ and $0<B<1, \eta(z)$ is manifestly positive real function. The conjugate of Eq. (A5) is

$$
\begin{gathered}
\int_{0}^{1} d z\left[-f\left|X_{z}\right|^{2}-\frac{2 b^{*}}{\sqrt{1-z}} X X_{z}^{*}+|X|^{2}\left\{\omega^{* 2} \eta(z)\right.\right. \\
\left.\left.-\frac{b^{*}}{2(1-z)^{3 / 2}}-\frac{l(l+1)}{4(1-z)^{3 / 2}}\right\}\right]=0 .
\end{gathered}
$$

The difference between Eqs. (A5) and Eq. (A6) is

$$
\begin{aligned}
& \int_{0}^{1} d z\left[-\frac{2 b}{\sqrt{1-z}} X^{*} X_{z}+\frac{2 b^{*}}{\sqrt{1-z}} X X_{z}^{*}\right. \\
& \left.+|X|^{2}\left\{\left(\omega^{2}-\omega^{* 2}\right) \eta(z)-\frac{\left(b-b^{*}\right)}{2(1-z)^{3 / 2}}\right\}\right]=0 .
\end{aligned}
$$

We integrate by parts the second term in above expression to get

$$
\begin{aligned}
& \int_{0}^{1} d z \frac{2 b^{*} X X_{z}^{*}}{\sqrt{1-z}}=-2 b^{*}\left|X\left(r_{0}\right)\right|^{2} \\
& -\int_{0}^{1} d z\left[\frac{2 b^{*} X^{*} X_{z}}{\sqrt{1-z}}+\frac{b^{*}|X|^{2}}{(1-z)^{3 / 2}}\right],
\end{aligned}
$$

where, $X\left(r_{0}\right)$ is the value of the function $X$ at the horizon. We write $\omega$ as $\omega=\omega_{R}+i \omega_{I}$. Then, one can simplify and get $b+b^{*}=-\frac{\omega_{I}}{r_{0}}(1-B)$ and $\omega^{2}-\omega^{* 2}=4 i \omega_{R} \omega_{I}$. Simplifying Eq. (A7) using Eq. (A8) and these results, one obtains

$$
\begin{aligned}
& \int_{0}^{1} d z \frac{(1-B) X^{*} X_{z}}{r_{0} \sqrt{1-z}}=\frac{b^{*}}{\omega_{I}}\left|X\left(r_{0}\right)\right|^{2} \\
& -\int_{0}^{1} d z|X|^{2}\left\{2 i \omega_{R} \eta(z)+\frac{(1-B)}{4 r_{0}(1-z)^{3 / 2}}\right\} .
\end{aligned}
$$

We use the above result to replace the second term in Eq. (A5). After a little simplification and rearrangement, one obtains

$$
\begin{aligned}
& \omega_{I} \int_{0}^{1} d z\left[f\left|X_{z}\right|^{2}+|X|^{2}\left\{\frac{l(l+1)}{4(1-z)^{3 / 2}}+|\omega|^{2} \eta(z)\right\}\right] \\
& =-\frac{|\omega|^{2}(1-B)}{2 r_{0}}\left|X\left(r_{0}\right)\right|^{2} .
\end{aligned}
$$

One observes that all terms in the integrand on the left hand side are manifestly positive for $0<z, B<1$. Then Eq. (A10) will be satisfied only if $\omega_{I}$ is negative. Hence all solutions must decay in time and this black hole with spherical horizon topology is stable against scalar perturbations.

\section{References}

1. B.P. Abbott, LIGO Scientific and Virgo Collaborations. Phys. Rev. Lett. 116(6), 061102 (2016). https://doi.org/10.1103/PhysRevLett. 116.061102. arXiv: 1602.03837 [gr-qc]

2. D. Birmingham, Phys. Rev. D 64, 064024 (2001). arXiv:hep-th/0101194

3. R. Aros, C. Martinez, R. Troncoso, J. Zanelli, Phys. Rev. D 67, 044014 (2003). arXiv:hep-th/0211024 
4. Ivo Sachs, Fortsch. Phys. 52, 667-671 (2004). arXiv:hep-th/0312287

5. O. Aharony, S.S. Gubser, J.M. Maldacena, H. Ooguri, Y. Oz, Phys. Rept 323, 183 (2000). https://doi.org/10.1016/ S0370-1573(99)00083-6. arXiv:hep-th/9905111

6. Gray T. Horowitz, Veronika E. Hubeny, Phys. Rev. D 62, 024027 (2000). arXiv:hep-th/9909056

7. D. Birmingham, I. Sachs, S.N. Solodukhin, Phys. Rev. Lett. 88, 151301 (2002). arXiv:hep-th/0112055

8. A. Nunez, A.O. Starinets, Phys. Rev. D 67, 124013 (2003). arXiv:hep-th/0302026

9. B. Wang, C.Y. Lin, E. Abdalla, Phys. Lett. B 481, 79-88 (2000). arXiv:hep-th/0003295

10. Dietmar Klemm, Alessio Marranib, Nicol'o Petria and Camilla Santolia. JHEP 09, 205 (2015). arXiv:1507.05553 [hep-th]

11. E.W. Leaver, Proc. R. Soc. Lond. A 402, 285-298 (1985)

12. E.W. Leaver, Phys. Rev. D 43, 1434 (1991)

13. Hisashi Onozawa, Takashi Mishima, Takashi Okamura, Hideki Ishihara, Phys. Rev. D 53, 7033-7040 (1996). arXiv:gr-qc/9603021

14. M. Richartz, Phys. Rev. D 93(6), 064062 (2016)

15. M. Abramowitz, I.A. Stegun, Handbook of mathematical functions with formulas, graphs, and mathematical tables (WileyInterscience, New York, 1972)

16. A. Lopez-Ortega, D. Mata-Pacheco. arXiv:1806.06547 [gr-qc]

17. G. Panotopoulos, Gen. Rel. Grav. 50(6), 59 (2018)
18. V. Cardoso, J.L. Costa, K. Destounis, P. Hintz, A. Jansen, Phys. Rev. Lett. 1203, 031103 (2018)

19. R.A. Janik, J. Jankowski, H. Soltanpanahi, JHEP 1606, 047 (2016)

20. T.R. Govindarajan, V. Suneeta, Class. Quant. Grav. 18, 265-276 (2001). arXiv:gr-qc/0007084

21. V. Cardoso, J.P.S. Lemos, Phys. Rev. D 63, 124015 (2001). arXiv:gr-qc/0101052

22. V. Cardoso, J.P.S. Lemos, Phys. Rev. D 64, 084017 (2001). arXiv:gr-qc/0105103

23. V. Cardoso, J.P.S. Lemos, Class. Quant. Grav. 18, 5257-5267 (2001). arXiv:gr-qc/0107098

24. R.A. Konoplya, Phys. Rev. D 66, 044009 (2002). arXiv:hep-th/0205142

25. A. Chowdhury, N. Banerjee. arXiv:1807.09559 [gr-qc]

26. V. Ferrari, B. Mashhoon, Phys. Rev. D 30, 295 (1984)

27. S. Dyatlov, M. Zworski, Phys. Rev. D 88, 084037 (2013)

28. I.G. Moss, J.P. Norman, Class. Quant. Grav. 19, 2323-2332 (2002). arXiv:gr-qc/0201016

29. Y. Kurita, M.A. Sakagami, Phys. Rev. D 67, 024003 (2003). arXiv:hep-th/0208063

30. E. Hawking, D. Page, Commun. Math. Phys. 87, 577 (1983)

31. P. Prasia, V.C. Kuriakose, Eur. Phys. J. C 77(1), 27 (2017)

32. F. Denef, S.A. Hartnoll, S. Sachdev, Phys. Rev. D 80, 126016 (2009)

33. P. Arnold, P. Szepietowski, D. Vaman, JHEP 1607, 032 (2016) 